\title{
Grand canonical Monte Carlo and modified singlet integral equations for the density profile of a Yukawa fluid near a planar wall
}

Douglas Henderson

Wilmer Olivares-Rivas

Leo Degreve

Jacqueline Quintana

Follow this and additional works at: https://scholarsarchive.byu.edu/facpub

Part of the Biochemistry Commons, and the Chemistry Commons

\section{Original Publication Citation}

Rivas, Wilmer O., Leo Degreve, Douglas Henderson, and Jacqueline Quintana."'Grand canonical Monte Carlo and modified singlet integral equations for the density profile of a Yukawa fluid near a planar wall." The Journal of Chemical Physics 16 (1997): 816

\section{BYU ScholarsArchive Citation}

Henderson, Douglas; Olivares-Rivas, Wilmer; Degreve, Leo; and Quintana, Jacqueline, "Grand canonical Monte Carlo and modified singlet integral equations for the density profile of a Yukawa fluid near a planar wall" (1997). Faculty Publications. 666.

https://scholarsarchive.byu.edu/facpub/666

This Peer-Reviewed Article is brought to you for free and open access by BYU ScholarsArchive. It has been accepted for inclusion in Faculty Publications by an authorized administrator of BYU ScholarsArchive. For more information, please contact ellen_amatangelo@byu.edu. 


\title{
Grand canonical Monte Carlo and modified singlet integral equations for the density profile of a Yukawa fluid near a planar wall
}

\author{
Wilmer Olivares-Rivas \\ Grupo de Química Téorica (QUIFFIS), Departamento de Química, Universidad de Los Andes, Mérida, \\ Venezuela \\ Léo Degrève \\ Grupo de Simulacão Molecular, Departamento de Química, Faculdade de Filosofia Ciencias e Letras, \\ Universidade de São Paulo, 14040-901 Ribeirão Preto, SP, Brazil \\ Douglas Henderson \\ Department of Chemistry and Biochemistry, Brigham Young University, Provo, Utah 84602-5700
}

Jacqueline Quintana

Instituto de Química, Universidad Nacional Autónoma de México (UNAM), México, DF 04510, Mexico

(Received 16 December 1996; accepted 30 January 1997)

Results for the density profile for Yukawa molecules near a hard wall and an exponential attractive wall are presented for Grand Canonical Monte Carlo (GCMC) simulations, for the singlet hypernetted chain (HNC) integral equation and for a modified version of the Lovett-Mou-BuffWertheim (LMBW-1) which uses the exact contact value theorem. The results of the standard singlet $\mathrm{HNC}$ are quite poor. If the LMBW equation is modified (but still using the bulk direct correlation function) the results at high temperature become reasonable. However, the results at low temperatures, close to the bulk coexistence curve, are only a partial improvement. The contact value of the density profile is now quite good but the profile has the incorrect oscillatory behavior predicted by the unmodified approximate equation. The GCMC simulation results show a clear effect of drying at low temperatures. (C) 1997 American Institute of Physics.

[S0021-9606(97)51817-4]

\section{INTRODUCTION}

The calculation of the density profile of an inhomogeneous fluid, through the use of an integral equation, can be performed using either the singlet Ornstein-Zernike (OZ1) equation $^{1,2}$

$$
h\left(z_{1}\right)=c\left(z_{1}\right)+\rho \int d \mathbf{r}_{2} h\left(z_{2}\right) c_{B}\left(R_{12}\right)
$$

or the pair Ornstein-Zernike (OZ2) equation, ${ }^{3}$

$$
\begin{aligned}
h\left(r_{12}, z_{1}, z_{2}\right)= & c\left(r_{12}, z_{1}, z_{2}\right) \\
& +\rho \int d \mathbf{r}_{3} \rho\left(z_{3}\right) h\left(r_{13}, z_{1}, z_{3}\right) c\left(r_{23}, z_{2}, z_{3}\right) .
\end{aligned}
$$

To yield a solution for either of the pair functions $h\left(r_{12}, z_{1}, z_{2}\right)$ and $c\left(r_{12}, z_{1}, z_{2}\right)$ or the singlet density profile, Eq. (1.2) must be supplemented by an additional relation between them. One useful relation is the Lovett-MouBuff-Wertheim (LMBW) relation ${ }^{4,5}$

$$
\frac{\partial \ln \rho\left(z_{1}\right)}{\partial z_{1}}=-\beta \frac{\partial V\left(z_{1}\right)}{\partial z_{1}}+\int d \mathbf{r}_{2} \frac{\partial \rho\left(z_{2}\right)}{\partial z_{2}} c\left(r_{12}, z_{1}, z_{2}\right) .
$$

In Eqs. (1.1) to (1.3), $\mathbf{r}_{i}$ is the position of molecule $i$ and $R_{12}=\left|r_{1}-r_{2}\right|$. Further, we have assumed for simplicity that the inhomogeneity is planar. Thus, $z_{i}$ is the perpendicular distance of molecule $i$ from the wall that produces the inho- mogeneity and $r_{12}=\left[R_{12}-\left(z_{1}-z_{2}\right)^{2}\right]^{1 / 2}$ is the projection of $R_{12}$ onto the plane of the inhomogeneity. The density profile is $\rho(z)=\rho[h(z)+1]$, where $\rho=\rho(\infty)$ is the bulk fluid density. The functions $h(z)$ and $h\left(r_{12}, z_{1}, z_{2}\right)$ are the singlet and pair total correlation functions, respectively, and $c(z)$ and $c\left(r_{12}, z_{1}, z_{2}\right)$ are the singlet and pair direct correlation functions, respectively. The function $c_{B}\left(R_{12}\right)=c\left(r_{12}, \infty, \infty\right)$, with $z_{1}-z_{2}$ finite, is the bulk pair direct correlation function which, in applications of Eq. (1.1), is assumed to be known. Finally $V(z)$ is the external potential, $\beta=1 / k T, k$ is Boltzmann constant and $\mathrm{T}$ the temperature.

To complete Eq. (1.1) or Eq. (1.2), a relation between $h$ and $c$ must be postulated. For Eq. (1.1) popular relations are the Percus-Yevick (PY) approximation

$$
h(z)-c(z)=[h(z)+1] \exp \{\beta V(z)\}-1,
$$

the hypernetted chain (HNC) approximation

$$
h(z)-c(z)=\ln [h(z)+1]+\beta V(z),
$$

and the mean spherical approximation (MSA)

$$
\begin{aligned}
& h(z)=-1, \quad z<\sigma / 2, \\
& c(z)=-\beta V(z), \quad z>\sigma / 2 .
\end{aligned}
$$

Equation (1.6) presumes that the molecules are excluded from the region $z<\sigma / 2$, which is appropriate if the inhomogeneity is due to a hard wall and is an exact statement of this presumed fact. Equation (1.7) is a linearization of Eq. (1.5). The PY, HNC and MSA approximations for the pair func- 
TABLE I. Values of the parameters $a, b, v,(p / \rho k T)_{E}$ and $\beta \mu^{\text {ex }}$ for a MSA Yukawa fluid.

\begin{tabular}{lrrrrr}
\hline \hline$k T / \epsilon$ & \multicolumn{1}{c}{$a$} & \multicolumn{1}{c}{$b$} & $v$ & $(p / \rho k T)_{E}$ & \multicolumn{1}{c}{$\beta \mu^{\mathrm{ex}}$} \\
\hline$\infty$ & 18.6502 & -19.1194 & 0.0000 & 5.7102 & 7.3593 \\
2.00 & 13.0556 & -17.6269 & 4.7964 & 2.9097 & 2.1638 \\
1.25 & 9.7372 & -16.7952 & 7.6892 & 1.2444 & -0.9418 \\
1.1 & 8.5403 & -16.1492 & 8.7449 & 0.6425 & -2.4248 \\
0.95 & 6.9757 & -16.1492 & 10.1379 & -0.1455 & -3.5469 \\
\hline \hline
\end{tabular}

tions in Eq. (1.2), are obtained from Eqs. (1.4) through (1.7) by replacing the singlet functions by the appropriate pair functions and replacing $V(z)$ by the intermolecular pair potential $u\left(R_{12}\right)$.

Interestingly, if $c\left(r_{12}, z_{1}, z_{2}\right)$ is replaced by $c_{B}\left(R_{12}\right)$, Eq. (1.3) becomes, after integration, Eq. (1.1) in the HNC approximation. In passing we note that observations such as this have sometimes lead to speculations that Eq. (1.1) is not exact and that $c_{B}\left(R_{12}\right)$ in Eq. (1.1) should be replaced by $c\left(r_{12}, z_{1}, z_{2}\right)$ to obtain an exact, or at least an improved equation. However, if the inhomogeneous $c\left(r_{12}, z_{1}, z_{2}\right)$ is used in Eq. (1.1), the first bridge diagram in $h(z)$ or $c(z)$ appears with coefficient of unity, instead of the correct coefficient of $1 / 2$.

\section{HARD WALL/YUKAWA SYSTEM}

We apply these integral equations to the Yukawa fluid of diameter $\sigma$ near a hard wall

$$
\begin{aligned}
& u(R)= \begin{cases}\infty, & R<\sigma \\
-\epsilon_{F} \sigma \exp \{-\lambda(R-\sigma) / \sigma\} / R, \quad R>\sigma,\end{cases} \\
& V(z)= \begin{cases}\infty, & z<\sigma / 2 \\
0, & z>\sigma / 2 .\end{cases}
\end{aligned}
$$

We use $\lambda=1.8$ since for this value the Yukawa fluid simulates argon reasonably well. ${ }^{6}$ The parameter $\epsilon_{F}$ is the depth of the fluid-fluid potential.

The Yukawa fluid pair potential does not appear in the OZ1 equation. All that is needed is the Yukawa fluid direct correlation function. It is particularly convenient to use the analytic MSA result, due to Waisman, ${ }^{7}$

$c_{B}(R)$

$$
=\left\{\begin{array}{l}
a+b R / \sigma+\frac{1}{2} \eta a R^{3} / \sigma+v \sigma(1-\exp \{-\lambda R / \sigma\}) / \lambda R, \\
R<\sigma \\
\beta \epsilon_{F} \sigma \exp \{-\lambda(R-\sigma) / \sigma\} / R, \\
\quad R>\sigma,
\end{array}\right.
$$

where $\eta=\pi \rho \sigma^{3} / 6$ and $a, b$ and $v$ are parameters which are defined implicitly and were obtained using the prescription of Hoye and Stell. ${ }^{8}$ In particular, $a=\beta(\partial p / \partial \rho)$. Some values of $a, b, v$ and $p / \rho k T$ (energy route) are given in Table I for $\rho \sigma^{3}=0.7$.

Using the HNC approximation, Eq. (1.1) becomes

$$
\begin{aligned}
\ln \left[h\left(z_{1}\right)+1\right]= & -\rho \int_{z_{1}}^{\infty} d z_{2} K_{B}\left(z_{2}\right)+\int_{\sigma / 2}^{\infty} d z_{2} \\
& \times\left[\rho\left(z_{2}\right)-\rho\right] K_{B}\left(\left|z_{1}-z_{2}\right|\right),
\end{aligned}
$$

where a hard wall, Eq. (2.2), has been assumed and

$$
K_{B}(x)=2 \pi \int_{x}^{\infty} c_{B}(R) R d R .
$$

Since $c_{B}(R)$ is analytic, an analytic expression for $K_{B}(x)$ results. Equation (2.4) yields

$$
\rho(\sigma / 2)=\rho[h(\sigma / 2)+1]=\frac{1}{2}(1+a),
$$

whereas the exact result is given by the contact value theorem for a hard wall

$$
\rho(\sigma / 2)=\beta p .
$$

Equation (2.4) is referred to as the singlet HNC (Ref. 9) or, when obtained through the direct method of Lozada-Cassou, ${ }^{2}$ as the three point extension HNC. ${ }^{10}$ It has been examined by several authors for hard spheres near hard walls. ${ }^{1,9,10}$ This is the high temperature limit of the system considered here. They found that, for that system, the results for the density profile are at least quantitatively reasonable. This is not unexpected. Comparison of Eqs. (2.6) and (2.7), using the values given in Table I, shows that the agreement is fairly good at high temperatures.

Here we are interested in lower temperatures. We know that Eq. (2.4) is deficient since comparison of Eq. (2.6) and Eq. (2.7), using the results of Table I, shows that Eq. (2.4) will yield increasingly poor results near the contact, $z_{1}=\sigma / 2$, as the temperature is decreased. However, a modified version of Eq. (2.4), which gives quite good results for the hard sphere/hard wall system, ${ }^{9}$ can be obtained easily. For a hard wall, the LMBW relation, Eq. (1.3), becomes

$$
\begin{aligned}
\frac{\partial \ln \rho\left(z_{1}\right)}{\partial z_{1}}= & \rho(\sigma / 2) K\left(z_{1}, \sigma / 2\right) \\
& +\int_{\sigma / 2}^{\infty} d z_{2} \frac{\partial \rho\left(z_{2}\right)}{\partial z_{2}} K\left(z_{1}, z_{2}\right),
\end{aligned}
$$

where

$$
K\left(z_{1}, z_{2}\right)=2 \pi \int_{0}^{\infty} \operatorname{drrc}\left(r, z_{1}, z_{2}\right) .
$$

We have already observed that if $c\left(r_{12}, z_{1}, z_{2}\right)$ is replaced by $c_{B}\left(R_{12}\right)$, Eq. (2.4) results. We shall therefore refer to this approximation as the LMBW-0 approximation. A modified version of the LMBW relation is obtained replacing $\rho(\sigma / 2)$ in Eq. (2.8) by $\beta p$. As long as no approximations are made, this replacement is formal. However if $c\left(r, z_{1}, z_{2}\right)$ is replaced by $c_{B}(R)$, we obtain after integration 


$$
\begin{aligned}
\ln \frac{\rho\left(z_{1}\right)}{\rho}= & {[\rho(\sigma / 2)-\rho-\beta p] \int_{z_{1}}^{\infty} d z_{2} K_{B}\left(z_{2}, \sigma / 2\right) } \\
& +\int_{\sigma / 2}^{\infty} d z_{2}\left[\rho\left(z_{2}\right)-\rho\right] K_{B}\left(\left|z_{1}-z_{2}\right|\right) .
\end{aligned}
$$

Equation (2.10) was proposed by Quintana et al. ${ }^{9}$ who found it to give improved results for the hard sphere/hard wall system. One purpose of this paper is to investigate its utility for a fluid which has attractive forces.

Equation (2.10) need not be restricted to hard walls. If the wall has an attractive tail, for instance an exponential tail,

$$
V(z)=\left\{\begin{array}{l}
\infty, \quad z<\sigma / 2 \\
-\epsilon_{W} \exp \{-\lambda(z-\sigma / 2) / \sigma\}, \quad z>\sigma / 2,
\end{array}\right.
$$

Eq. (2.7) becomes

$$
\rho(\sigma / 2)=\beta p+\int_{\sigma / 2}^{\infty} d z \frac{\partial V(z)}{\partial z} \rho(z),
$$

where $V(z)$ is the external wall/fluid potential for $\mathrm{z}>\sigma / 2$. In which case the modified singlet $\mathrm{HNC}$ equation is

$$
\begin{aligned}
\ln \frac{\rho\left(z_{1}\right)}{\rho}= & {\left[\rho(\sigma / 2)-\rho-\beta p-\beta \int_{0}^{\infty} d z \frac{\partial V(z)}{\partial z} \rho(z)\right] } \\
& \times \int_{z_{1}}^{\infty} d z_{2} K_{B}\left(z_{2}, \sigma / 2\right) \\
& +\int_{\sigma / 2}^{\infty} d z_{2}\left[\rho\left(z_{2}\right)-\rho\right] K_{B}\left(\left|z_{1}-z_{2}\right|\right)-\beta V\left(z_{1}\right) .
\end{aligned}
$$

Since this equation is identical to the modified LMBW equation, Eq. (2.8) together with Eq. (2.12), when $c\left(r_{12}, z_{1}, z_{2}\right)$ is replaced by $c_{B}\left(R_{12}\right)$, we shall refer to Eq. (2.13) as the LMBW-1 approximation.

\section{RESULTS}

The advantage of the Yukawa fluid is the existence of an analytic expression for $c_{B}\left(R_{12}\right)$. One disadvantage is the fact that there are no computer simulation results available for comparison. We have, therefore, carried out Grand Canonical Monte Carlo (GCMC) simulations for the system defined by the potential given in Eqs. (2.1) and (2.11).

The simulations were realized through alternating canonical and grand canonical Monte Carlo simulations ${ }^{11-13}$ at constant temperature $T$, volume $V$ and chemical potential $\mu$. The excess chemical $\mu^{\mathrm{ex}}$ was obtained in the mean spherical approximation and checked by the Grand Canonical Monte Carlo simulations. Each complete canonical cycle was alternated with one attempt for the creation/destruction of one molecule. The usual canonical and Grand Canonical Monte Carlo simulations methods were applied.

The number of complete cycles used during the equilibration phase varied from 5000 to 100000 while the data were acquired during 100000 or 200000 cycles. The simulated systems were constituted by two parallel walls distant by $10 \sigma$. The remaining edges of the simulation box were

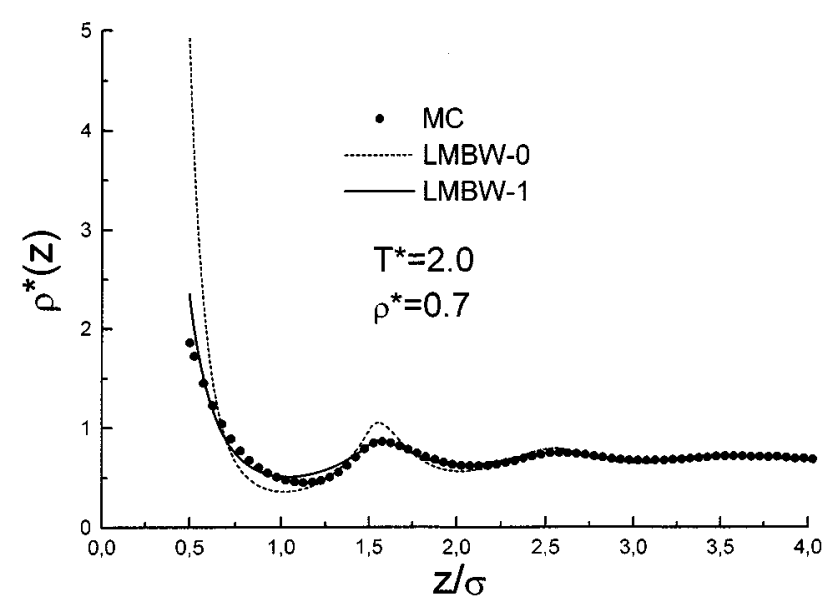

FIG. 1. The reduced particle density profile for a Yukawa fluid near a hard wall, for a reduced temperature $T^{*}=2.0$. The filled dots are our GCMC results, the dashed (LMBW-0) and full lines (LMBW-1) are the unmodified and modified approximate LMBW integral equations, respectively. All the results are for a reduced bulk density $\rho^{*}=0.7$.

both equal to $9 \sigma$. These dimensions were chosen larger than the distance where correlations are detected in the Yukawa systems so that we can accept that the surface structures on the walls distant by $10 \sigma$ are independent and equal to the structures that would be observed on infinitely distant walls. The usual periodic boundary conditions and minimum image conventions were applied in the directions parallel to the walls.

As expected, the results are very similar to those for a Lennard-Jones fluid near a wall studied by Plischke and Henderson $^{3}$ using the OZ2 equation, in the PY approximation and the simulations of Abraham ${ }^{14}$ and Sullivan et al. ${ }^{15}$

In Figs. (1) to (3) we show results for GCMC for a reduced density $\rho^{*}=0.7$ and several values of the reduced temperature $T^{*}=k T / \epsilon_{F}$ close to, but above, the coexistence vapor-liquid curve. ${ }^{16}$ In Fig. (1) we present the results for the

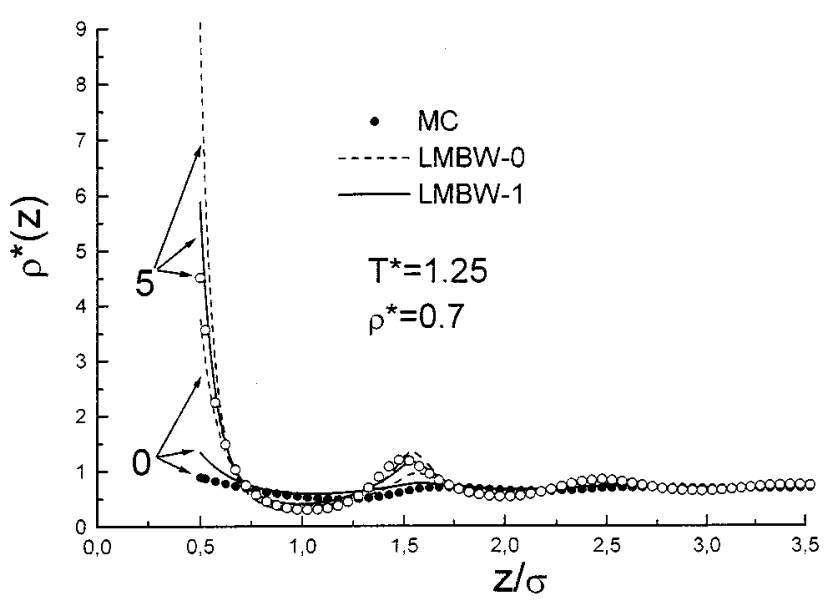

FIG. 2. Reduced density profile for a Yukawa fluid near a hard and an attractive Yukawa wall at $T^{*}=1.25$. As indicated, the upper set is for a ratio $\epsilon_{W} / \epsilon_{F}=5$, the lower set is for a hard wall, $\epsilon_{W} / \epsilon_{F}=0$. The open and full dots are the GCMC results. The integral equations are labeled as in Fig. (1). 


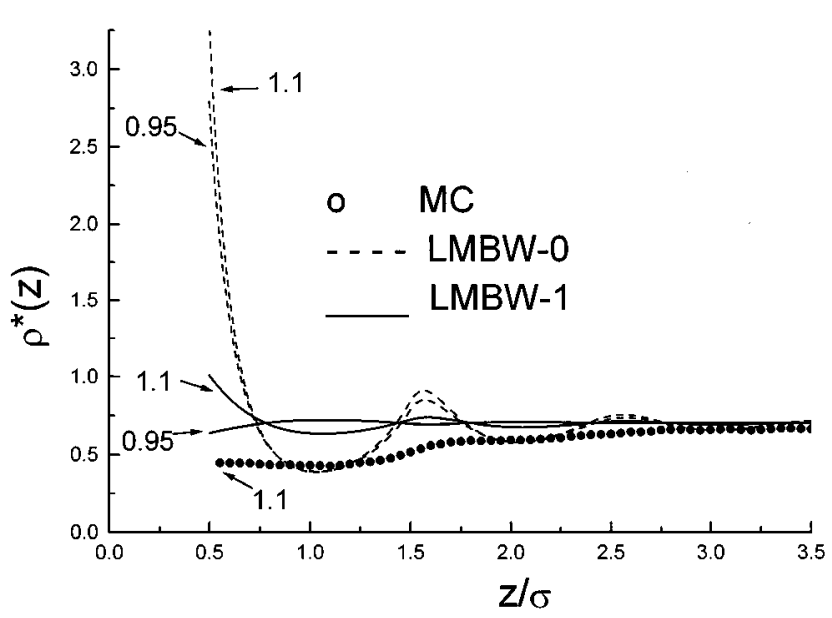

FIG. 3. Density profiles of a Yukawa fluid near a hard wall for two low temperatures, $T^{*}=1.1$ and $T^{*}=0.95$, showing an effect of drying. The curves are labeled as in Fig. (1).

reduced density profiles, $\rho^{*}(z)$ vs $z / \sigma$, for $T^{*}=2$. This temperature is high enough for the density profile to resemble the hard wall/hard sphere structure as obtained previously by Plischke and Henderson ${ }^{16}$ and Quintana et al. ${ }^{7}$ The GCMC results present strong oscillations and show that the particles are pushed against the wall.

As the temperature is reduced, as shown in Figs. (2) and (3) for $T^{*}=1.25$ and $T^{*}=1.1$, the oscillations become smaller and the curves become monotonic. Further, the contact value, $\rho(\sigma / 2)$, decreases, as would be expected from Eq. (2.7), and the slope of the density profile at contact changes its sign, showing the effect of a drying transition. That is, the particles are pushed away from the hard wall. This effect is much the same as that reported for a Lennard-Jones fluid near a hard wall. ${ }^{3,14,15}$

In Fig. (1) we also show the results for the LMBW equation, with the MSA $c_{B}(R)$. Here the original equation, Eq. (2.8) or equivalently Eq. (2.4), is labeled LMBW-0. The LMBW approximation, modified using the contact value theorem but still using the bulk MSA $c_{B}(R)$, Eq. (2.8) with Eq. (2.12) or equivalently Eq. (2.13), is labeled LMBW-1. At high temperatures, the results are identical to the earlier results. ${ }^{9}$ That is, the high temperature LMBW-1 are quite reasonable. However, as shown in Fig. (1) for $T^{*}=2.0$, the high temperature profiles obtained using the unmodified equation LMBW-0, or equivalently the OZ1 HNC/MSA equation, are much too large at contact.

As we decrease the temperature, as shown in Fig. (2) for $T^{*}=1.25$ the unmodified equation retains its oscillatory behavior with much too high contact values, as compared to the GCMC data (filled circles) while the modified LMBW-1 equation becomes smoother in reasonable agreement with the GCMC data.

In Fig. (2) we compare the results for a hard wall, $\boldsymbol{\epsilon}_{W} / \boldsymbol{\epsilon}_{F}=0$, and an attractive wall with $\boldsymbol{\epsilon}_{W} / \boldsymbol{\epsilon}_{F}=5$ and the same range parameter $\lambda=1.8$. As expected, we observe that, as the wall becomes attractive, the oscillations in the structure of the fluid are enhanced giving rise to the layering of the particles in the vicinity of the wall. The LMBW-1 modified equation still gives good agreement with the GCMC computer simulations, while the LMBW-0 unmodified equation gives a contact value almost twice the GCMC value.

For lower temperatures, approaching the liquid-vapor transition temperature, the discrepancy between both theories and the simulation data becomes important. As shown in Fig. (3), again the unmodified LMBW-0 results at contact are too large and the profile is highly oscillatory while they should be monotonic as predicted by the GCMC simulation. This incorrect behavior has already been observed by Sullivan et al. ${ }^{15}$ and Plischke and Henderson ${ }^{3}$ for Lennard-Jones molecules near a hard wall. The modified LMBW-1 results for $\rho(z)$ are a modest improvement in as much $\rho(\sigma / 2)$ is small. The slope at contact changes from negative to positive values at a reduced temperature between 1 and 0.9. Regrettably, the LMBW-1 curves give the incorrect oscillatory behavior seen in the unmodified LMBW-0.

\section{SUMMARY}

We have calculated $\rho(z)$ for a Yukawa fluid near a hard or a Yukawa wall from GCMC simulations and from the modified LMBW equation with the homogeneous direct correlation function replaced by $c_{B}\left(R_{12}\right)$. This latter approximate LMBW equation is a singlet theory closely related to the hypernetted chain approximation.

The results of this approximate LMBW equation, referred to as LMBW-1, are quite good at high temperatures for both hard and attractive walls but are only partially satisfactory at low temperatures in the vicinity of the liquidvapor fluid bulk transition. The contact value is good but the profile is oscillatory and not monotonic as expected and predicted by our GCMC simulation results.

When compared to the simulation data then, the modified LMBW equation appears in general to be a good improvement over the original unmodified approximate LMBW equation or, equivalently, the standard singlet $\mathrm{OZ} 1 \mathrm{HNC}$ integral equation.

We expect the modified LMBW equation to be satisfactory when used with the OZ2 relation since even with the unmodified equation the results are already quite good for Lennard-Jones molecules. ${ }^{14,18}$

\section{ACKNOWLEDGMENTS}

The authors are grateful to program PLAN III and Centro de Cálculo Científico (CeCalCULA) of the University of Los Andes, Venezuela, to the Conselho Nacional de Desenvolvimento Científico e Tecnológico and to the Fundação de Amparo à Pesquisa do Estado de São Paulo, Brazil. J. Q. is thankful to CONACYT, México, for financial support. The support of the National Science Foundation, Grants CTS94023584 and CHE96-01971 is acknowledged with thanks.

\footnotetext{
${ }^{1}$ D. Henderson, F. F. Abraham, and J. A. Barker, Mol. Phys. 31, 1291 (1976)

${ }^{2}$ M. Lozada-Cassou, J. Chem. Phys. 75, 1412 (1981).

${ }^{3}$ M. Plischke and D. Henderson, J. Chem. Phys. 84, 2846 (1986).

${ }^{4}$ R. Lovett, C. Y. Mou, and F. P. Buff, J. Chem. Phys. 65, 2377 (1976).
} 
${ }^{5}$ M. Wertheim, J. Chem. Phys. 65, 570 (1976).

${ }^{6}$ D. Henderson, E. Waisman, L. Lebowitz, and L. Blum, Mol. Phys. 35, 241 (1978)

${ }^{7}$ E. Waisman, Mol. Phys. 25, 45 (1973).

${ }^{8}$ J. S. Høye and G. Stell, Mol. Phys. 32, 195 (1976).

${ }^{9}$ J. Quintana, D. Henderson, and M. Plischke, J. Phys. Chem. 93, 4304 (1989).

${ }^{10}$ J. Alexandre, M. Lozada-Cassou, and L. Degrève, Mol. Phys. 88, 1388 (1996).

${ }^{11}$ N. Metropolis, A. W. Rosenbluth, M. N. Rosenbluth, A. H. Teller, and E. Teller, J. Chem. Phys. 21, 1087 (1953).
${ }^{12}$ M. P. Allen and D. J. Tildesley, Computer Simulation of Liquids (Clarendon, Oxford, 1987).

${ }^{13}$ G. Cicotti, D. Frenkel, and I. R. McDonald, Simulation of Liquids and Solids. Molecular Dynamics, and Monte Carlo Methods in Statistical Mechanics (North-Holland, Amsterdam, 1990).

${ }^{14}$ F. F. Abraham, J. Chem. Phys. 68, 3715 (1978).

${ }^{15}$ D. Sullivan and G. Stell, J. Chem. Phys. 69, 5450 (1978); D. E. Sullivan, D. Levesque, and J. J. Weis, ibid. 72, 1170 (1980).

${ }^{16}$ E. Lomba and N. G. Almarza, J. Chem. 100, 8367 (1994).

${ }^{17}$ Plischke and D. Henderson. Proc. R. Soc. London., Ser. A 404, 323 (1986)

${ }^{18}$ Plischke and D. Henderson, J. Chem. Phys. 93, 4489 (1990). 